Intensity-based dual model method for generation of synthetic

CT images from standard T2-weighted MR images Generalized technique for four different MR scanners

Koivula, Lauri

2017

Koivula , L , Kapanen , M , Seppälä , T , Collan , J , Dowling , J A , Greer , P B , Gustafsson , C , Gunnlaugsson , A, Olsson, L E , Wee , L \& Korhonen , J 2017 , ' Intensity-based dual model method for generation of synthetic CT images from standard T2-weighted MR images - Generalized technique for four different MR scanners ', Radiotherapy and Oncology , vol. 125 , no. 3 , pp. 411-419 . https://doi.org/10.1016/j.radonc.2017.10.011

http://hdl.handle.net/10138/298623

https://doi.org/10.1016/j.radonc.2017.10.011

publishedVersion

Downloaded from Helda, University of Helsinki institutional repository.

This is an electronic reprint of the original article.

This reprint may differ from the original in pagination and typographic detail.

Please cite the original version. 
Imaging

\title{
Intensity-based dual model method for generation of synthetic CT images from standard T2-weighted MR images - Generalized technique for four different MR scanners
}

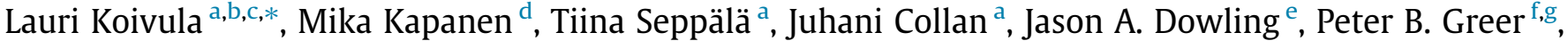 \\ Christian Gustafsson ${ }^{\text {h,i }}$, Adalsteinn Gunnlaugsson ${ }^{\mathrm{h}}$, Lars E. Olsson ${ }^{\mathrm{i}, \mathrm{j}}$, Leonard Wee ${ }^{\mathrm{k}, \mathrm{l}}$, Juha Korhonen ${ }^{\mathrm{a}, \mathrm{c}, \mathrm{m}, \mathrm{n}}$

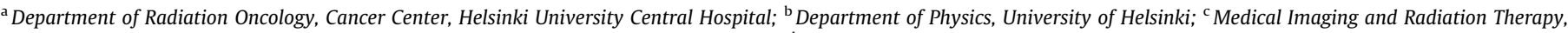

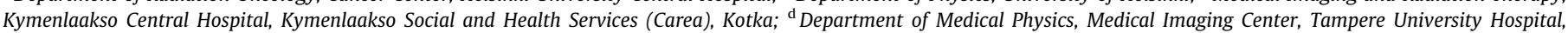

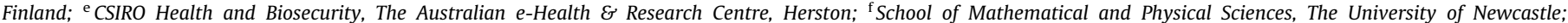

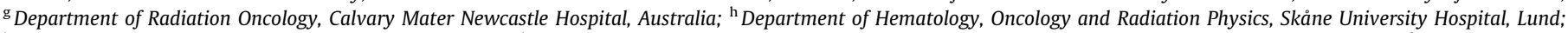

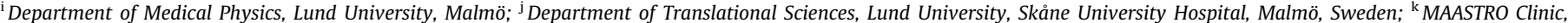

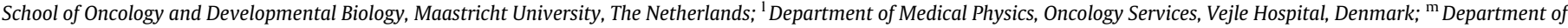 \\ Radiology; and ${ }^{\mathrm{n}}$ Department of Nuclear Medicine, Helsinki University Central Hospital, Finland
}

\section{A R T I C L E I N F O}

\section{Article history:}

Received 28 May 2017

Received in revised form 9 October 2017

Accepted 10 October 2017

Available online 30 October 2017

\section{Keywords:}

MRI-only

Radiation therapy

Synthetic CT images

\begin{abstract}
A B S T R A C T
Background and purpose: Recent studies have shown that it is possible to conduct entire radiotherapy treatment planning (RTP) workflow using only MR images. This study aims to develop a generalized intensity-based method to generate synthetic CT ( $\mathrm{sCT}$ ) images from standard T2-weighted (T2 $\left.2_{\mathrm{w}}\right) \mathrm{MR}$ images of the pelvis.

Materials and methods: This study developed a generalized dual model HU conversion method to convert standard T2 $2_{\mathrm{w}}$ MR image intensity values to synthetic HU values, separately inside and outside of atlassegmented bone volume contour. The method was developed and evaluated with 20 and 35 prostate cancer patients, respectively. MR images with scanning sequences in clinical use were acquired with four different MR scanners of three vendors.

Results: For the generated synthetic CT ( $\mathrm{SCT}$ ) images of the 35 prostate patients, the mean (and maximal) $\mathrm{HU}$ differences in soft and bony tissue volumes were $16 \pm 6 \mathrm{HUs}$ ( $34 \mathrm{HUs}$ ) and $-46 \pm 56 \mathrm{HUs}$ (181 HUs), respectively, against the true CT images. The average of the PTV mean dose difference in sCTs compared to those in true CTs was $-0.6 \pm 0.4 \%(-1.3 \%)$.

Conclusions: The study provides a generalized method for $\mathrm{sCT}$ creation from standard $\mathrm{T}_{\mathrm{w}}$ images of the pelvis. The method produced clinically acceptable dose calculation results for all the included scanners and MR sequences.
\end{abstract}

(C) 2017 Elsevier B.V. All rights reserved. Radiotherapy and Oncology 125 (2017) 411-419
Magnetic resonance imaging (MRI) has gained a major role in radiotherapy target and organ-at-risk (OAR) delineation due to its high soft tissue contrast and versatile nature [1-3]. The current standard practice is to apply both MRI and computed tomography (CT) for the radiotherapy treatment planning (RTP) workflow MRI for delineation, and CT for dose calculation and imageguided radiotherapy (IGRT). This workflow is prone to possible systematic errors when MR and CT images are co-registered for transferring delineated contours to images used in dose calculation and IGRT [4,5]. Omitting the CT scan and using an MRI-only

\footnotetext{
* Corresponding author at: Department of Radiation Oncology, Cancer Center Helsinki University Central Hospital, POB-180, 00029 HUS Helsinki, Finland.

E-mail address: lauri.koivula@helsinki.fi (L. Koivula).
}

approach would minimize these systematic errors [6] and reduce the number of planning scans hence increasing patient comfort, saving time, and reducing need for clinical resources.

MR images do not contain electron density (ED) information and therefore cannot be directly utilized for dose calculations. The MRI-only workflow incorporates so-called synthetic CT (sCT) images in which Hounsfield unit (HU) values are reconstructed using only the original MR image. Recent studies have shown that it is possible to perform entire RTP workflow, including image guidance, using only MR images [1,7-13].

Several methods have been published for SCT image generation [14]. Three main categories are: use of bulk densities [15,16], atlasbased MR image registration to a model CT image $[17,18]$ and voxel-based intensity conversions [9,19]. The bulk density 
approach uses uniform HU values for large segmented volumes e.g. whole body or bones. Atlas-based SCT construction utilizes coregistered MR- and CT-images to transfer the $\mathrm{HU}$ values to an MR image, whereas voxel-based methods rely on the original MR image information (voxel intensity value or spatial location, etc.) to transform the MR image intensity values to HU values. Some sCT construction methods include combinations of the categories. The dual model HU conversion method transforms MR image intensity values of T1-weighted in-phase MR image directly to predefined HU values separately inside and outside of an atlas-based auto-contoured bone volume [9,20-22]. The method has been applied for clinical MRI-only RTP protocol at the Cancer Center of Helsinki University Central Hospital. Since 2012, the clinic has treated nearly 400 prostate cancer patients with the MRI-only workflow $[1,9,10,23]$.

Direct MR intensity to HU value conversion is fast and easy to adopt with various software. Conversion algorithm is iterationfree, which enables HU conversion in roughly $30 \mathrm{~s}$ with our current software and hardware setup. There is a potential to further optimize the conversion to reach sub-second timescale.

Previous SCT studies have mainly evaluated the feasibility of each method for only one MR scanner with particular imaging parameters. In practice, manufacturer and scanner differences, magnetic field strength, image acquisition and coil differences will inevitably result in variations in MRI signals. Recently, Persson et al. have studied prostate RTP dose calculation accuracy of atlas-based sCTs produced via cloud-based conversion system [24]. They presented a mean dose difference of $0.2 \pm 0.4 \%$ in the target with 145 patients with four different MR scanners. The primary aim of this study is to develop a generalized intensity-based conversion method functioning with standard $\mathrm{T} 2$-weighted $\left(\mathrm{T} 2 \mathrm{w}_{\mathrm{w}}\right)$ images acquired with scanners of different vendors, and with variety of imaging parameters. The generalized model is intended to act as a backbone for adopting intensity-based conversion workflow for a specific scanner. MR images were obtained from four different clinics using their local imaging workflows. The work aims to construct a robust clinically available workflow to be employed with generic medical image processing software to automatically perform both steps of the sCT generation method (bone segmentation and intensity conversion). The quality of the produced SCT images should be independent from inter-patient, -scanner or clinic differences; e.g. patient size, absolute MR image intensity values or coil positioning. The main focus was on prostate cancer, for which $\mathrm{T} 22_{\mathrm{w}}$ images are already used as standard target delineation images, and thus potentially no extra sequences would be needed for the MRI-only protocol.

\section{Materials and methods}

\section{Scanners and patient cohort}

Four scanners from three different vendors - two with $1.5 \mathrm{~T}$ and two with $3.0 \mathrm{~T}$ field strengths - were included in the study (Table 1). Scanners are referred to by their arithmetic numeration throughout the article. Four clinics from Finland, Sweden, Denmark, and Australia provided MR images for this study, all with their individual choice of set-up, coils, sequences, and workflows. Images obtained for the study were conventional T2-weighted sequences in clinical use without any modifications made for this study. Images were collected with large field-of-view (FOV), ranging from $43 \mathrm{~cm}$ to $50 \mathrm{~cm}$, to cover the whole-body contour. Vendor-based gradient nonlinearity and intensity inhomogeneity corrections were applied for the images. The study included a total of 20 patients for model creation and 35 patients for model evaluation. All the patient data were collected retrospectively and this study had no effect on their treatment.

\section{Synthetic CT generation method}

The dual model HU conversion method was used as a base for the method presented in this study. The model has been described earlier in detail $[9,10,20-22]$. In brief, the model consists of autosegmentation of the bone volume followed by conversion of MR image intensities to $\mathrm{HU}$ values separately within and outside of the bone contour. The dual model method features polynomial and threshold-based conversions within and outside of bone segment, respectively. Initially a fourth-degree polynomial fit was used for the bone volume MR image intensity values, accompanied with fine tuning of the conversion curve for minimizing differences on the whole HU scale. Polynomial was converted to step-wise function with twenty segments to speed up the HU conversion software. Soft tissue conversion relies on known HU values of adipose tissue and muscle, and measured variance of the MR intensities in these tissues. The MR intensity values in between the two tissue classes were linearly interpolated to HUs with step-wise functions. In this study, corresponding MRI and CT intensity values were collected using 2.5 and $5.0 \mathrm{~mm}$ diameter data collection volume-of-interests (VOI) for the bony tissue and soft tissue classes, respectively [9]. A rigid registration between the MR and CT image volumes was made based on mutual information [9].

As the dual model method uses absolute MR image intensity values, the feasibility of sCT generation is dependent on the overall intensity level of the MR image. MR image intensity levels vary

Table 1

Parameters of the used MR-sequences.

\begin{tabular}{|c|c|c|c|c|}
\hline & Scanner 1 & Scanner 2 & Scanner 3 & Scanner 4 \\
\hline Scanner model & GE Optima $1.5 \mathrm{~T}$ & Philips Ingenia $1.5 \mathrm{~T}$ & Siemens Skyra 3.0 T & GE Discovery $3.0 \mathrm{~T}$ \\
\hline Sequence type & FSE T2 ${ }_{w} 3 D$ & $\mathrm{TSE} \mathrm{T} 2_{\mathrm{w}} 2 \mathrm{D}$ & FSE T2 $2_{w} 3 \mathrm{D}$ & FSE T2 $2_{w} 2 \mathrm{D}$ \\
\hline Echo time & $97 \mathrm{~ms}$ & $100 \mathrm{~ms}$ & $102 \mathrm{~ms}$ & $102 \mathrm{~ms}$ \\
\hline Repetition time & $1900 \mathrm{~ms}$ & $9392 \mathrm{~ms}$ & $1200 \mathrm{~ms}$ & $15,000 \mathrm{~ms}$ \\
\hline Echo train length (ETL) & 84 & 25 & 80 & 15 \\
\hline Bandwidth & $355 \mathrm{~Hz} /$ pix & $203 \mathrm{~Hz} /$ pix & $781 \mathrm{~Hz} /$ pix & $390 \mathrm{~Hz} /$ pix \\
\hline Acquisition matrix & $448 \times 448$ & $500 \times 500$ & $256 \times 256$ & $640 \times 512$ \\
\hline Reconstruction matrix & $512 \times 512$ & $768 \times 768$ & $256 \times 256$ & $1024 \times 1024^{*}$ \\
\hline Flip angle & 90 & 90 & 135 & 130 \\
\hline Number of slices & $72-124$ & 60 & 128 & 88 \\
\hline Field-of-view & $450 \times 450 \mathrm{~mm}^{2}$ & $500 \times 500 \mathrm{~mm}^{2}$ & $430 \times 430 \mathrm{~mm}^{2}$ & $448 \times 448 \mathrm{~mm}^{2}$ \\
\hline Pixel size & $0.9 \mathrm{~mm}$ & $0.7 \mathrm{~mm}$ & $1.6 \mathrm{~mm}$ & $0.4 \mathrm{~mm}(0.9 \mathrm{~mm})$ \\
\hline Slice thickness & $2.4 \mathrm{~mm}$ & $2.0 \mathrm{~mm}$ & $1.6 \mathrm{~mm}$ & $2.5 \mathrm{~mm}$ \\
\hline Distance between slice centers & $1.2 \mathrm{~mm}$ & $3.0 \mathrm{~mm}$ & $1.6 \mathrm{~mm}$ & $2.5 \mathrm{~mm}$ \\
\hline Patients for model creation & 5 & 5 & 5 & 5 \\
\hline Patients for model evaluation & 10 & 5 & 10 & 10 \\
\hline
\end{tabular}

Reconstruction matrix was reduced to $512 \times 512$ for data processing and sCT creation. 
Table 2

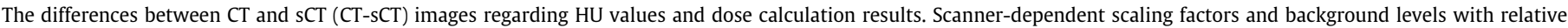
dose difference in OARs are presented. "Dose comparison results against standard CT images for scanner-specific conversion for scanner 3 are displayed in parentheses.

\begin{tabular}{|c|c|c|c|c|c|}
\hline Scanner model & $\begin{array}{l}\text { Scanner } 1 \\
\text { GE Optima } 1.5 \mathrm{~T}\end{array}$ & $\begin{array}{l}\text { Scanner } 2 \\
\text { Philips Ingenia } 1.5 \mathrm{~T}\end{array}$ & $\begin{array}{l}\text { Scanner } 3^{*} \\
\text { Siemens Skyra } 3.0 \mathrm{~T}\end{array}$ & $\begin{array}{l}\text { Scanner } 4 \\
\text { GE Discovery } 3.0 \mathrm{~T}\end{array}$ & All patients \\
\hline Number of patients & 10 & 5 & 10 & 10 & 35 \\
\hline Mean diff. \pm SD Soft tissue (HUs) & $16 \pm 7$ & $20 \pm 8$ & $18 \pm 3$ & $13 \pm 6$ & $16 \pm 6$ \\
\hline Bone (HUs) & $-79 \pm 41$ & $-91 \pm 32$ & $14 \pm 22$ & $-51 \pm 1$ & $-46 \pm 56$ \\
\hline Dose Mean diff. \pm SD (\%) & $-0.5 \pm 0.4$ & $-0.5 \pm 0.2$ & $-0.9 \pm 0.2(-0.2 \pm 0.1)$ & $-0.4 \pm 0.2$ & $-0.6 \pm 0.4$ \\
\hline V5\% & $-0.3 \pm 0.4$ & $-0.3 \pm 0.2$ & $-0.8 \pm 0.2(-0.1 \pm 0.1)$ & $-0.3 \pm 0.2$ & $-0.4 \pm 0.4$ \\
\hline V95\% & $-0.6 \pm 0.3$ & $-0.5 \pm 0.2$ & $-1.0 \pm 0.2(-0.4 \pm 0.1)$ & $-0.5 \pm 0.2$ & $-0.7 \pm 0.3$ \\
\hline Scaling factor (mean \& SD) & $0.47 \pm 0.06$ & $2.96 \pm 0.15$ & $1.27 \pm 0.12$ & $0.21 \pm 0.02$ & - \\
\hline Background reduction (value \& SD) & $28 \pm 3$ & $6 \pm 1$ & $0 \pm 0$ & $374 \pm 54$ & - \\
\hline \multicolumn{6}{|l|}{$\begin{array}{l}\text { OAR dose difference (\%) } \\
\text { Mean dose }\end{array}$} \\
\hline Rectum & $-0.1 \pm 0.2$ & $-0.2 \pm 0.1$ & $-0.3 \pm 0.05(-0.1 \pm 0.04)$ & $-0.1 \pm 0.1$ & $-0.19 \pm 0.13$ \\
\hline Bladder & $-0.2 \pm 0.1$ & $-0.2 \pm 0.03$ & $-0.3 \pm 0.1(-0.2 \pm 0.1)$ & $-0.2 \pm 0.1$ & $-0.24 \pm 0.09$ \\
\hline Femoral head (left) & $-0.1 \pm 0.1$ & $-0.1 \pm 0.1$ & $-0.3 \pm 0.1(-0.1 \pm 0.1)$ & $-0.2 \pm 0.1$ & $-0.19 \pm 0.11$ \\
\hline Femoral head (right) & $-0.2 \pm 0.1$ & $-0.1 \pm 0.04$ & $-0.3 \pm 0.1(-0.1 \pm 0.1)$ & $-0.2 \pm 0.1$ & $-0.19 \pm 0.11$ \\
\hline \multicolumn{6}{|l|}{$D V H(\%)$} \\
\hline Rectum (V40\%) & $-0.1 \pm 0.4$ & $-0.3 \pm 0.2$ & $-0.3 \pm 0.1(-0.2 \pm 0.1)$ & $-0.3 \pm 0.2$ & $-0.24 \pm 0.23$ \\
\hline Bladder (V40\%) & $-0.2 \pm 0.1$ & $-0.2 \pm 0.1$ & $-0.2 \pm 0.1(-0.1 \pm 0.1)$ & $-0.1 \pm 0.1$ & $-0.17 \pm 0.11$ \\
\hline Femoral head (left, V20\%) & $-0.5 \pm 0.5$ & $-0.7 \pm 0.7$ & $-1.4 \pm 0.8(-0.5 \pm 0.5)$ & $-0.7 \pm 0.4$ & $-0.84 \pm 0.73$ \\
\hline Femoral head (right, V20\%) & $-0.7 \pm 0.3$ & $-0.5 \pm 0.3$ & $-1.2 \pm 0.8(-0.5 \pm 0.3)$ & $-0.8 \pm 0.9$ & $-0.83 \pm 0.68$ \\
\hline
\end{tabular}

between scanner models and manufacturers - even when using similar pulse sequence $[25,26]$. There is no standardization of MR intensities as in CT numbers [27].

Therefore, in this study, we developed a generalized method which is not affected by the absolute MR intensity levels. The generalized method incorporates a scaling of the original MR intensities to a base level, followed by the MR intensity value to HU value conversion with the dual model method.

The scaling of the intensity levels of MR images was performed by removing the background noise signal level - measured from cortical bone near isocenter using 20 VOIs with $2.5 \mathrm{~mm}$ diameter - and scaling all MR image intensity values linearly according to the absolute MR image intensity of the adipose tissue near the isocenter - collected with 20 VOIs with $5 \mathrm{~mm}$ diameter. The process is described in Eq. (1):

$I_{\text {final }}=\left(I_{\text {original }}-b\right) \times \frac{k_{\text {average }}}{k}$

where $I$ is the intensity of a given voxel for a patient before and after scaling, $b$ is the background noise signal value, $k_{\text {average }}$ represents the average base value of the adipose tissue of all the 20 patients in model creation group, and $k$ is the intensity value of adipose tissue of a particular patient. The adoption of adipose tissue MR intensity values near the isocenter was considered optimal to minimize influence of image intensity inhomogeneities (intra-patient variations) on conversion accuracy, and to define general intensity level of a particular image (inter-patient variations). The base value to which all images were scaled was an intensity value of 200 . This value was an approximate mean intensity level of the adipose tissue near the isocenter of all 20 images.

These scaling values $\left(k_{\text {average }} / k\right.$ ) varied considerably among platforms and the factors that were used are presented in Table 2. Conversion curves for the model were created according to the scaled data collection VOIs and were divided into twenty-one and nine intensity intervals for $\mathrm{HU}$ value conversion for bony and soft tissue volumes, respectively, seen in the Fig. 1c) and d) as step functions. Additionally, the study included scanner-specific conversion models, and evaluated potential improvements on SCT construction by using those instead of applying the developed general model.

Atlas-based auto-segmentation for the bone contours was conducted using commercial image processing software MIM v6.5.5 (MIM Software, Inc., Cleveland, OH, USA). Five patients of the scanner 1 patient group were incorporated in the bone atlas \#1.
Auto-segmentation method of 5/5 was used; five patients were used in the auto-segmentation process of the total five patients present in the atlas. Scanner 1 bone atlas \# 1 was also used for contouring scanner 2 images. Original scanner 2 images were scaled to match average intensity level of scanner 1 images and then autocontoured using bone atlas \#1 with scanner 1 images. This was done to demonstrate the feasibility of using standard $\mathrm{T} 2{ }_{\mathrm{w}}$ images across scanner platforms for the atlas-based auto-segmentation. Bone contours for scanner 3 patients were contoured with the multi-atlas/local weighted voting auto-segmentation method applied by Dowling et al [11]. Scanner 4 images were contoured similarly to scanner 1 , as $5 / 5$ method was used with scanner 4 images in the atlas. Different contouring approaches were selected to point out that any well-functioning auto-segmentation workflow is compatible with the generalized dual model method.

No manual contouring was done after auto-segmentation for evaluating feasibility of the fast, clinical approach of the segmentation. Previous studies have shown that, the automatic atlas-based bone segmentation enables precise sCT generation and accurate dose calculation in the pelvic region $[11,18](0.1 \pm 0.1 \%$ dose differences in the prostate PTV compared to those with manually precisely contoured bones [21]). The exterior body contour of the CT image was used in the SCT instead of the MR image body contour to minimize the dose calculation differences arising from altered posture between $C T$ and MR imaging. The time frame between $\mathrm{CT}$ and MR acquisitions varied from minutes to days among the different clinics, creating inevitable changes to patient anatomy and skin position. Prior et al [28] have concluded that ignoring body outline changes can result up to several percent dose difference between CT and SCT images. Additionally, one of the MR scanners was not equipped with a flat table top. The presented workflow of MR intensity scaling and dual model HU value conversion was then repeated for the 35 prostate cancer patients in the evaluation group using the model created from the group of 20 different patients. Conversion time from MR image intensity values to $\mathrm{HU}$ values was approximately $30 \mathrm{~s}$ for each patient with standard quad-core desktop computer.

\section{Evaluation of sCT image quality and dose calculation accuracy}

Resulting HU values were compared against the CT image HU values by calculating the mean values for the soft and bony tissue volumes, and calculating the difference as CT-sCT. Treatment 

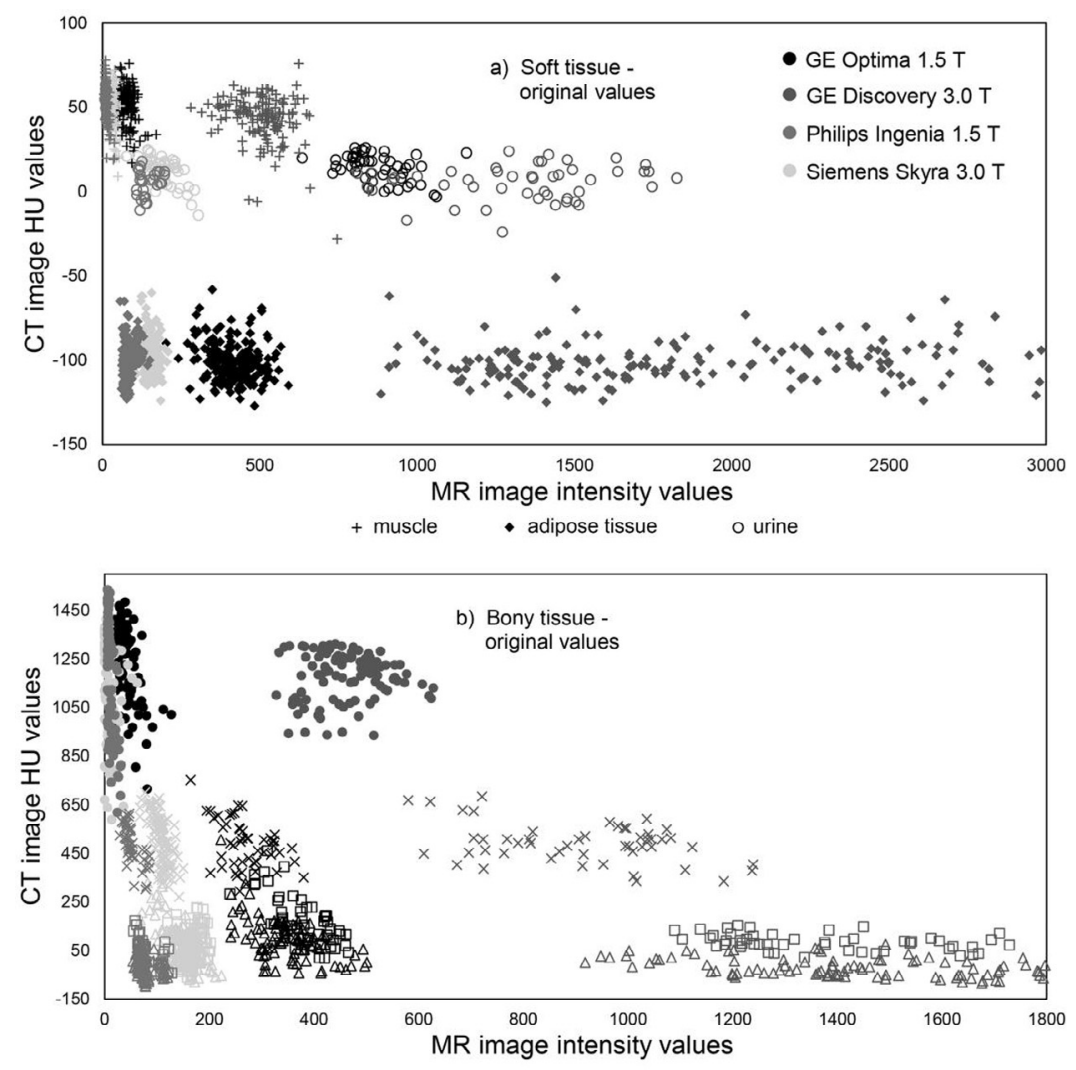

- cortical bone $x$ dense spongy bone $\square$ low density spongy bone $\Delta$ bone marrow
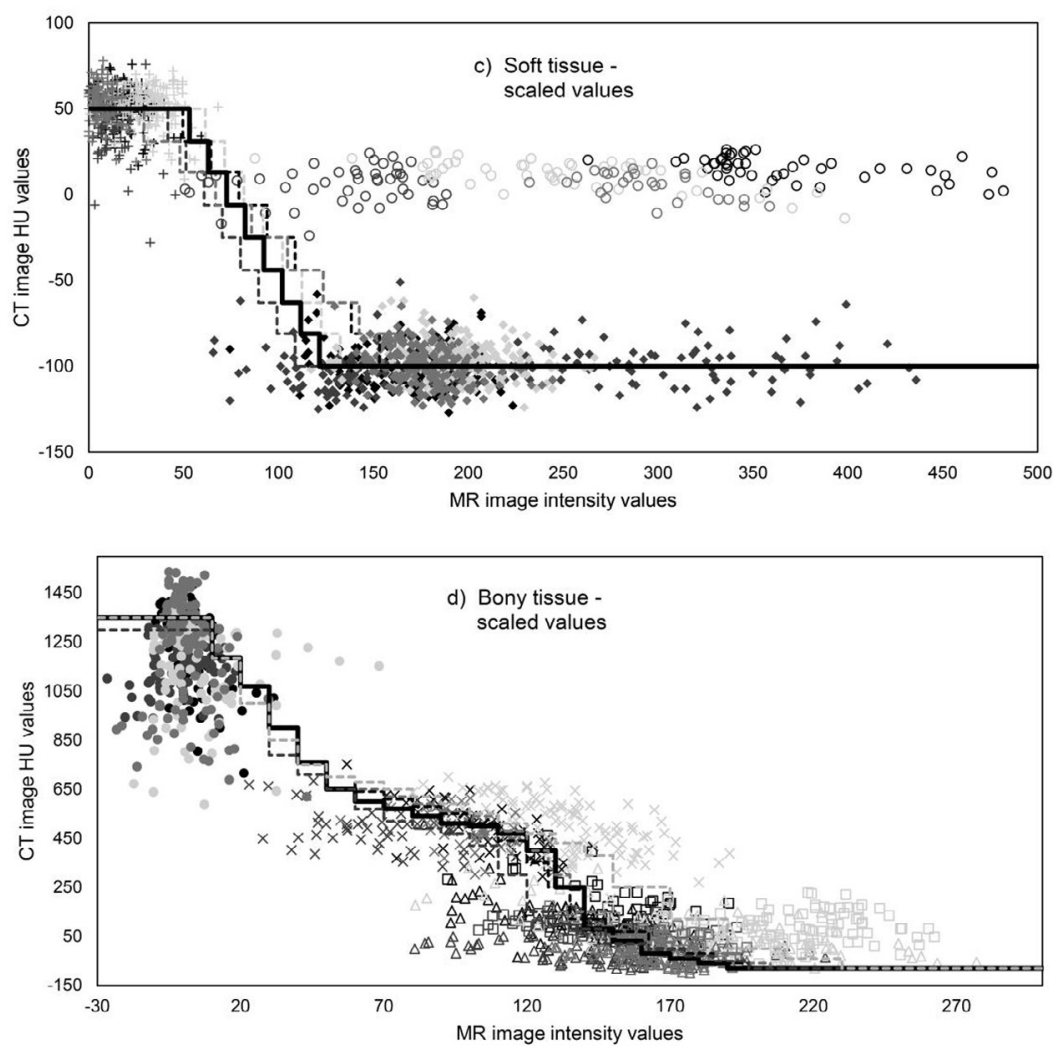

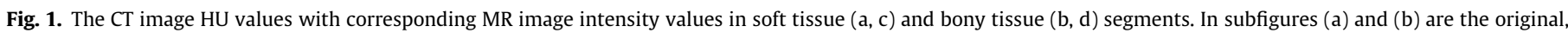

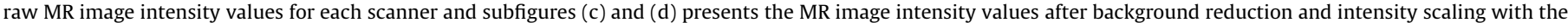
step functions of the conversions overlaid (thick line represents the general model and dashed lines correspond to the scanner-specific conversion curves). 
planning was conducted with full-arc volumetric modulated arc therapy (VMAT) using $10 \mathrm{MV}$ photons. Plans were optimized and calculated on the SCT images and then copied to CT images and re-calculated. Dose calculation was conducted using the anisotropic analytical algorithm (AAA 13.6.23, Varian Medical Systems, Palo Alto, USA) with grid size of $0.1 \mathrm{~cm}$. Dose comparisons were conducted by calculating differences in dose volume histogram (DVH) parameters between sCT and CT for the PTV (mean, V5, V95), OARs (rectum \& bladder [mean, V40\%] and femoral heads [mean, V20\%]). Contouring of the OARs was done on the MR image and then copied to the CT image - this workflow enables a better approximation of the dose differences in DVH analysis considering the OAR volume variation e.g. bladder filling. Analysis of variance (ANOVA) was conducted with SPSS (IBM Corp. Released 2013. IBM SPSS Statistics for Windows, Version 22.0. Armonk, NY: IBM Corp.) to study the differences between scanners.

\section{Results}

Fig. 1 presents CT image $\mathrm{HU}$ values with corresponding MR image intensity values from the data collection VOIs of the 20patient model creation group. Variation of original MR intensity values between the scanners can be seen in Fig. 1a) and b) for soft and bony tissues, respectively. Reduced variance after background reduction and intensity scaling is presented in Fig. 1c) and d). Soft tissue conversion incorporated muscle and adipose tissue HU values but omitted urine due to partial overlap with adipose tissue on the MR intensity scale with two sequences, hence urine was converted mainly to adipose tissue HU values, see Fig. 1c). Scanner-specific conversion curves in Fig. 1c) and 1d) provide comparison to the average conversion curve used for sCT creation.

Fig. 2 shows example images of the generated sCTs with corresponding MR and CT images accompanied by dose difference maps. Fig. 2e-h) shows example sCTs constructed by the generalized model, and Fig. 2o) presents a scanner-specific sCT (Scanner 3). The variation of the MR intensity values is demonstrated by scanner-specific intensity windows used in image reconstruction for visualization purposes. Table 2 presents results of the HU value and dose calculation comparisons between $\mathrm{CT}$ and SCT images of the 35-patient evaluation group. Mean whole soft tissue volume HU differences (CT-sCT) ranged from 13 to 20 HUs between scanners and mean whole bony tissue volume HU differences from -91 to 14 HUs. The largest average HU value difference was 34 HUs in soft tissue and 181 HUs in bony tissue segment. Intensity value scaling factors ranged from 0.18 to 3.17 in all scanners, but within each scanner the largest variation remained within $24 \%$ or lower compared to the mean scaling value of that scanner. The average of the PTV mean dose difference for all 35 patients in the evaluation group was $-0.6 \%$ with $\mathrm{SD}$ of $0.4 \%$ (range: $-0.1 \%$ to $-1.3 \%$ ), and DVH point differences for V5 and V95 were $-0.4 \pm$ $0.4 \%$ and $-0.7 \pm 0.3 \%$, respectively. The mean PTV dose difference for scanner-specific sCTs was $-0.2 \pm 0.1 \%$ with DVH point differences of $-0.1 \pm 0.1 \%$ and $-0.4 \pm 0.1 \%$ for V5 and V95, respectively. The largest individual patient dose difference for the mean PTV dose was $-1.3 \%$ and the largest single-voxel point dose difference

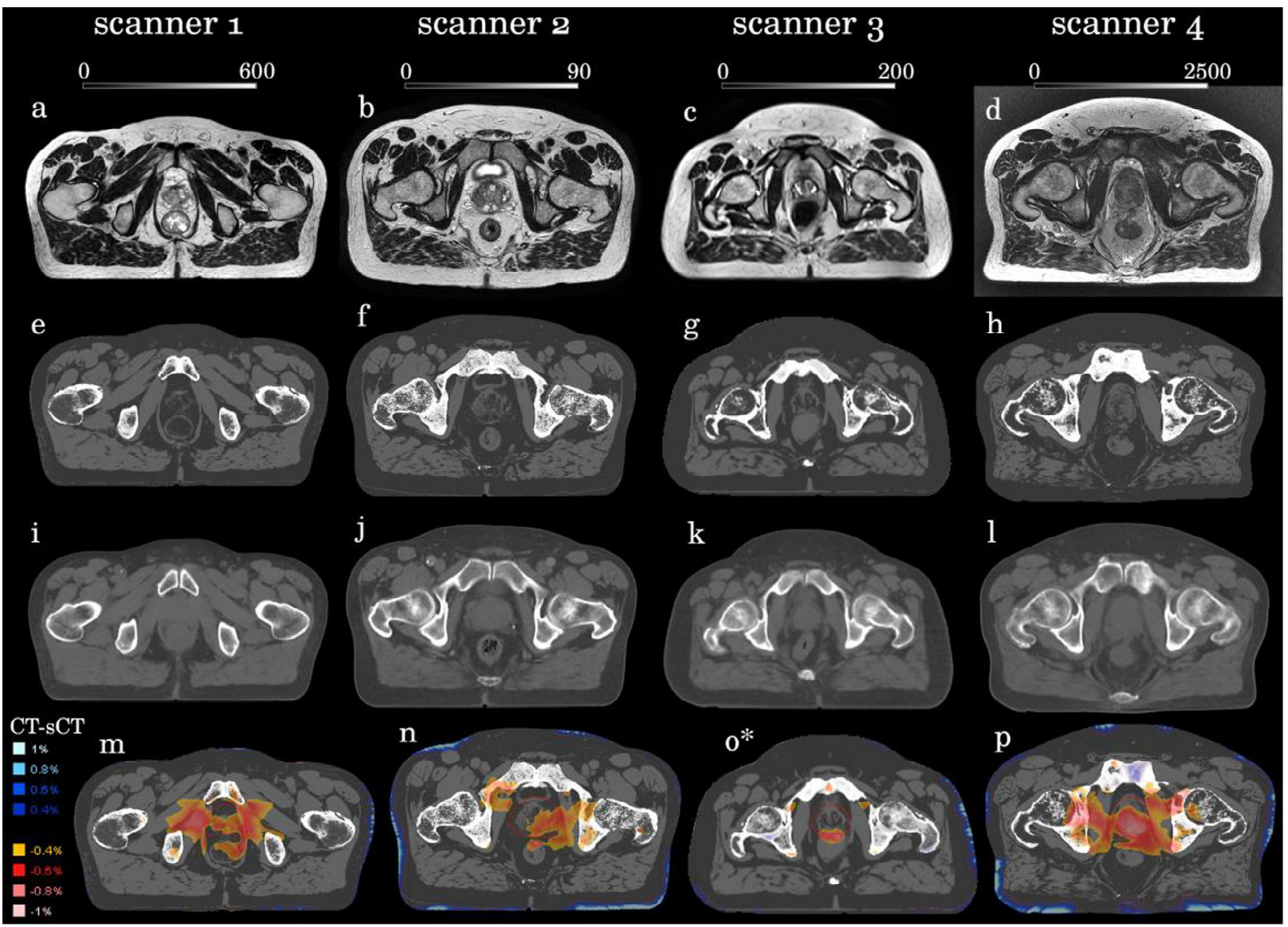

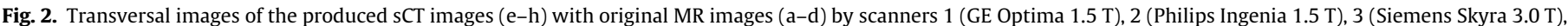

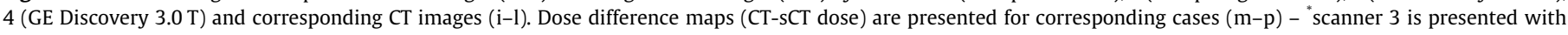

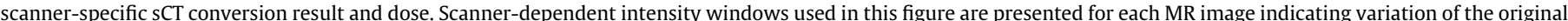

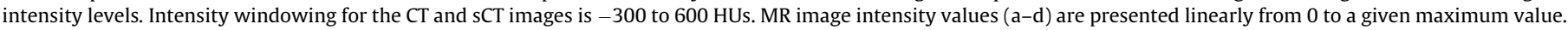
Due to this and differences in vendor-specific image reconstruction, the high background noise in the air is clearly visualized in the image $\mathrm{d}$. 
was $-1.7 \%,-1.9 \%,-1.9 \%$, and $-1.8 \%$ for Scanners $1,2,3$, and 4 , respectively. Dose comparison of the generalized sCTs for OAR volume mean dose differences resulted in $-0.2 \pm 0.1 \%$ difference for all specified structures (rectum, bladder, left and right femoral head) with average DVH point differences smaller than $1 \%$. Comprehensive OAR dose calculation results can be found in Table 2 . Fig. 3 presents orthogonal reconstructions of the $\mathrm{CT}(\mathrm{a}-\mathrm{c})$ images, scanner-specific conversion sCT (d-f) results with corresponding generalized SCT $(\mathrm{g}-\mathrm{i})$ - Dose comparison maps of a scannerspecific and generalized method against the $\mathrm{CT}$ image are found in subfigures $(\mathrm{j}-\mathrm{l})$ and $(\mathrm{m}-\mathrm{o})$, respectively.

ANOVA-analysis of the dose difference results for PTV mean doses shows that there is a significant $(p=0.025)$ variation between the scanners when using the generalized model
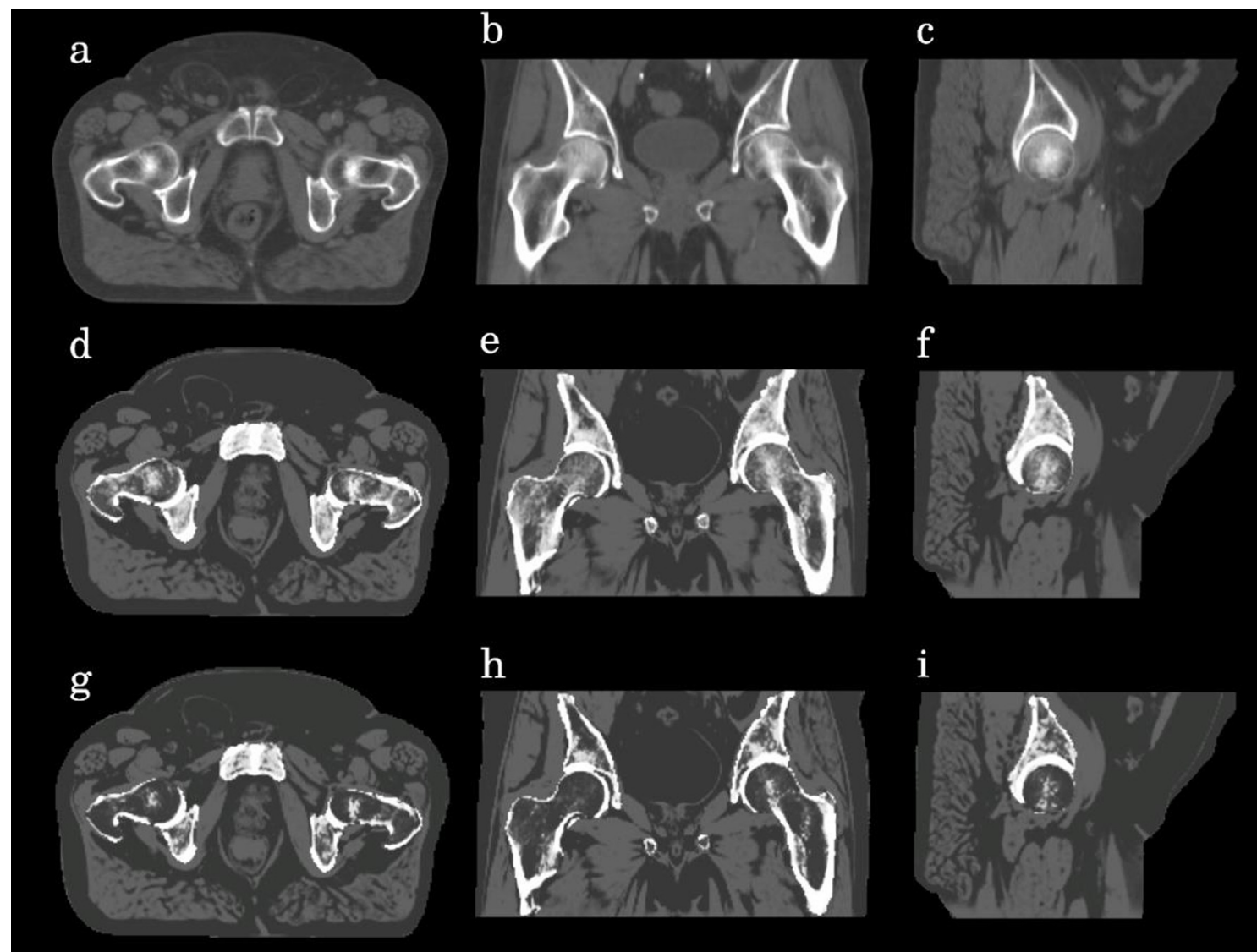

i
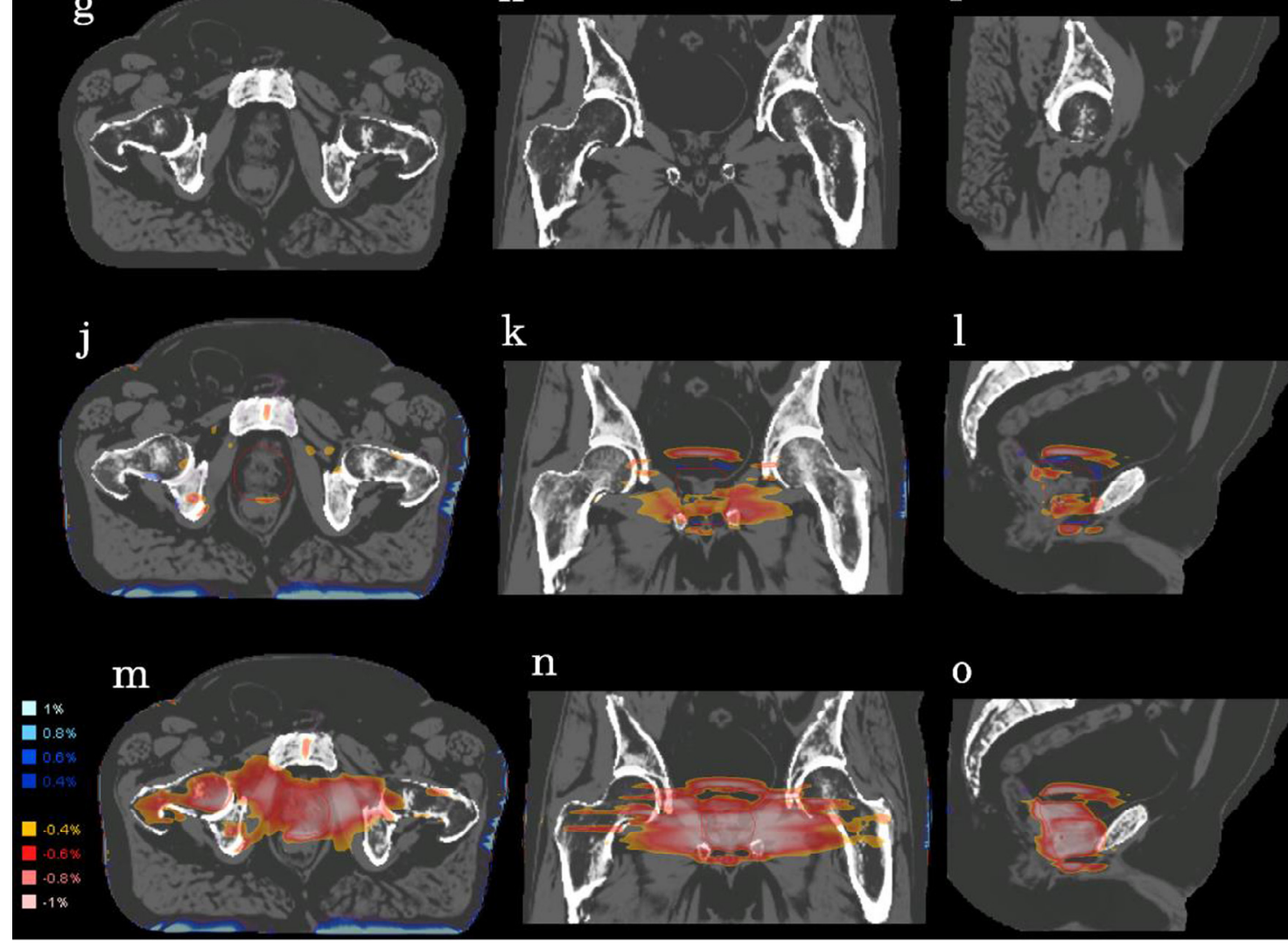

$\mathbf{n}$
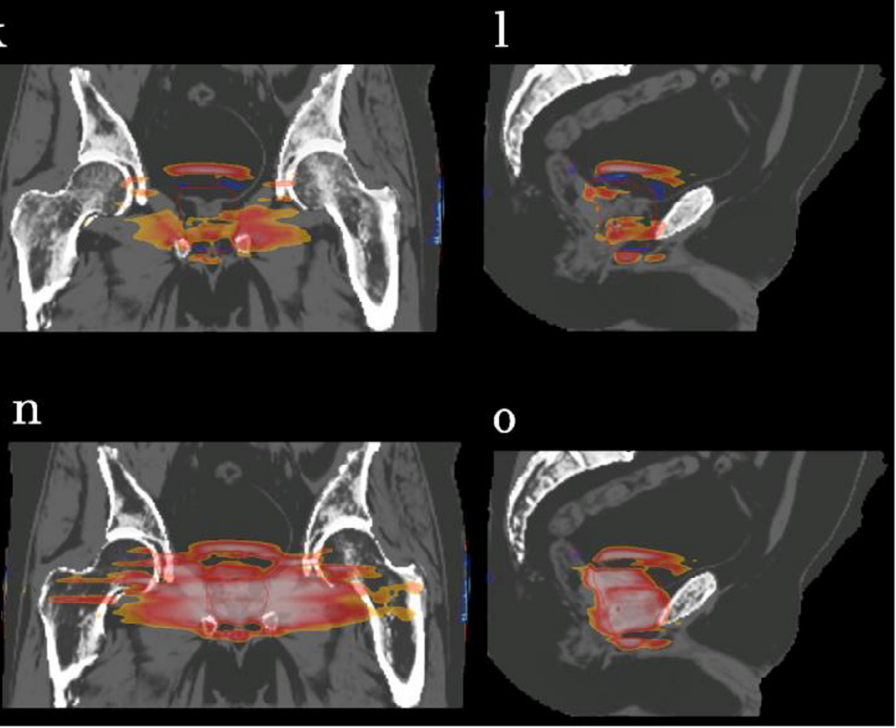

O

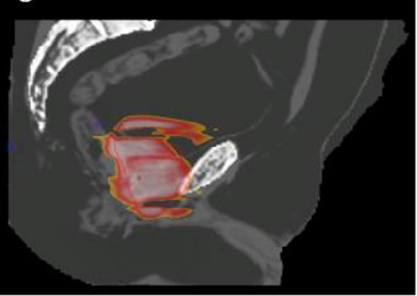

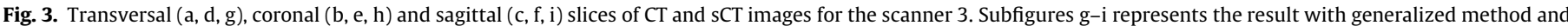

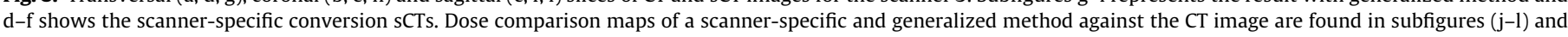

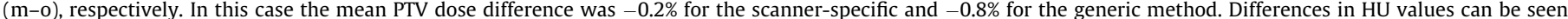

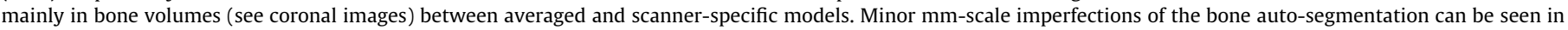
some bone edges (e.g. acetabulum). 
Table 3

Statistical analysis of the PTV mean dose differences between scanners with least significant difference (LSD) for the generalized model, p-values $<0.05$ are presented in bold.

\begin{tabular}{lllll}
\hline \multicolumn{5}{l}{$p$-Values of the scanner-to-scanner comparison } \\
\hline Scanners & 1 & 2 & 3 & 4 \\
\hline 1 & & 0.911 & $\mathbf{0 . 0 1 2}$ & 0.946 \\
2 & 0.911 & & $\mathbf{0 . 0 2 8}$ & 0.956 \\
3 & $\mathbf{0 . 0 1 2}$ & $\mathbf{0 . 0 2 8}$ & & $\mathbf{0 . 0 1 0}$ \\
4 & 0.946 & 0.956 & $\mathbf{0 . 0 1 0}$ & \\
\hline
\end{tabular}

Comparison between the scanners using least significance difference (LSD) reveals that scanner 3 sCTs varies significantly from the other three scanners - results are presented in Table 3. No significant differences were found between scanners 1,2 , and 4 .

\section{Discussion}

This study presented an automatic intensity-based SCT construction method for standard $\mathrm{T} 2{ }_{\mathrm{w}}$ images of the pelvis. The method was tested for multiple MRI platforms with variable imaging parameters, thus demonstrating the generalizability and robustness of the method for MRI-only RTP. The produced heterogeneous SCTs enabled clinically acceptable dose calculation accuracy. Overall dosimetric agreement in the prostate PTV was -0.6 $\pm 0.4 \%$ [range: -0.1 to $-1.3 \%$ ]. This is a comparable result with other previously published high quality sCTs [9-11,21,24,29-31]. Persson et al. [24] have presented mean PTV dose difference of $0.2 \pm 0.4 \%$ [range: 1.3 to $-0.7 \%$ ] without body outline correction and approximately $0.1 \pm 0.2 \%$ [range: 0.5 to $-0.6 \%$ ] with body contour correction. Dowling et al. [11] presented PTV DVH V50\% -0.5 $\pm 1.1 \%$ [quartiles $(25,75 \%) 0.0$ to $-1.4 \%$ ]. Korhonen et al [9] have previously shown a PTV DVH V50\% difference of $0.3 \pm 0.2 \%$ [range: $0.0-0.8 \%$.

The presented generalized conversion method provided extensive functionality despite the variation between different clinic's scanning workflows and MR images. The HU accuracy in the generated SCTs was roughly at a similar level to previously developed SCT methods. However, this generalized method included higher HU uncertainties compared to methods developed particularly for a specific sequence $[9,11,18,32,19,22]$. Scanner-specific conversion curves for SCT creation resulted in smaller mean PTV dose differences $(-0.9 \pm 0.2 \%$ vs. $-0.2 \pm 0.1 \%)$ for the Scanner 3 images. Generalized model provided an average PTV dose difference of $-0.5 \%$ or smaller in sCTs for all other scanners. All four scanners resulted in a PTV mean dose difference smaller than $1.3 \%$ with the generic method demonstrating the clinical feasibility - with scanner-specific conversion method the dose difference approach negligible values. The generalized method presents a model and an example of the HU conversion workflow for standard T2weighted MR images that can easily be adopted in other clinics. The collection of scanner-specific MR intensity values is encouraged when commissioning the MRI-only conversion protocol. Variation between scanner 3 and the other scanners was caused mainly by the MR intensity variation of the spongy bone. As the generalized model uses averages of multiple scanners, it cannot account for any exceptional variations from one scanner and intensity level. Precise determination of the accuracy of the SCT conversion is difficult due to other confounding factors that influence the dose differences including anatomical differences between consecutive scans [28] and the inherent HU value uncertainty in CT images (e.g. HU accuracy, HU-to-ED calibration, artifacts) [33,34].

The generalized method produced a small systematic error in the mean difference of the soft tissue HU values and in the mean PTV doses between CT and SCT images. The sCT images presented systematically lower soft tissue HUs with respectively higher dose in the target region, due to lower attenuation of the photon beam. As the applied RT plan was a full-arc VMAT, the HU value accuracy of the soft tissue volume has a relatively high effect on the dose distribution compared to plans containing mainly lateral beams through the femoral heads. There are several reasons for the observed results. As the method converts MR intensity values directly to HUs, soft tissue structures with high $\mathrm{HU}(0-50)$ and high MR signal are intrinsically misrepresented as adipose tissue. Such structures are for example bulbus penis, some blood vessels and nerves, seminal vesicles, and malignant prostate tissue with addition to all tissues containing liquid in a macroscopic scale. Higher HU values in bony tissue might be explained by the simplicity of the utilized polynomial fitting for the data. Furthermore, the fitting does not evaluate volumetric differences between bone structures (cortical bone, spongy bone, bone marrow) but includes only the intensity value data for the conversion model generation.

Fig. 1d) shows the largest variation of the conversion curves on the HU scale of 100-600 HUs, which corresponds mainly to the spongy bone volume. Correspondingly, the largest difference on the dose distribution of the OARs can be found in the femoral heads - an average difference of DVH point V20\% for all four scanners was $-0.8 \pm 0.7 \%$ and for scanner $3-1.3 \pm 0.8 \%$. The scanner-specific sCT reconstruction for scanner 3 resulted in smaller error and variation of the dose in femoral heads (average of $-0.5 \pm 0.4 \%$ ) as well in other OARs and PTV.

The MR image intensity inhomogeneity and functionality of the HU conversion could have a substantial effect on the dose distribution [8], as femoral heads incorporate a relatively large volume near the prostate. Thus, future studies could aim to further improve the model especially for spongy bones. Difference between sCTs with scanner-specific or generalized model for scanner 3 can be seen in the coronal images of the Fig. 3. Conversion of the soft tissue volume is almost invariant of the conversion method, but a noticeable difference is present in some parts of the bone volume.

The conversion method did not incorporate urine in the predefined $\mathrm{HU}$ values as there was an overlap of the signals for scanner 3 and 4, and it would have mislabeled large volumes of adipose tissue as water, even though sequences used with scanners 1 and 2 would have enabled the separation of adipose tissue and urine on the MRI intensity scale. Tuning the sequence parameters, e.g. the flip angle and echo train length (ETL), might enable better separation between different tissue classes on the MR image and hence result in more accurate heterogeneous SCT image. Including urine/water $\mathrm{HU}$ values in the conversion method would enable the conversion of high signal MRI voxels to water equivalent instead of adipose tissue, hence improving the overall HU equivalency. Precise conversion of urine for sCTs is not essential for MRI-only RTP of prostate cancer patients.

Homogeneity of MR image intensity level across the whole patient body volume is advantageous to obtain high quality intensity-based SCT conversion. The MR image inhomogeneity correction algorithms are of particular value. Such algorithms are standard in MR platforms and commercial image processing software $[35,36]$.

The presented method relies only on the absolute values of the MR images, so possible changes in intensity homogeneity and image artifacts could affect the resulting sCT image. Additionally, the selected background reduction and scaling factor values inherently influence the assigned HU values. Further validation and verification processes could assist in developing the most optimal method for the scaling factor selection for the implementation into the clinical MRI-only workflow.

Geometric distortions can restrict the use of MRI in RT. Geometrical distortions stemming from system related effects such 
as gradient non-linearity or $\mathrm{B}_{0}$ field inhomogeneities should be evaluated before introducing a clinical MRI-only workflow [37]. Additional artifacts can derive from patient induced distortions, such as susceptibility and chemical shift. Previous studies have shown that with geometric accuracy better than $2 \mathrm{~mm}$ within the entire FOV it is possible to achieve clinically feasible dose calculation accuracy for MRI-only RTP [21,23,38-41]. The geometric accuracy of the applied MR platforms was sufficient for MRI-only RT $[10,11,21,23,37]$.

An MRI-only approach is feasible and utilized internationally in few clinics for radiotherapy of the prostate employing either commercial or in-house sCT techniques. This study further advances and support future research and clinical implementation of MRIonly RTP by presenting a robust and practical synthetic CT conversion workflow for standard T2-weighted MR images.

\section{Conclusions}

This study introduced a generalized dual model HU conversion method to obtain high quality sCTs from standard T2-weighted MR images. The method was shown to be suitable for images acquired with different MR platforms of different vendors. The work presented has demonstrated that using the obtained sCTs it is possible to reach clinically feasible dose calculation accuracy for MRI-only RT of prostate cancer patients. The generalized conversion workflow serves as the core component enabling commissioning of MRI-only workflow in a particular clinic. The generalized method produced sCT images with clinically feasible dose calculation accuracy despite of inter-patient, -scanner and -clinic differences. The sCTs with scanner-specific conversion workflow presented similar dose distribution as in standard CT images. Fine tuning of the MR sequence parameters and $\mathrm{HU}$ conversion model could further improve the sCT quality and dose calculation accuracy.

\section{Conflict of interest}

ChG and LEO acknowledge the funding from the Swedish Innovation Agency (Gentle Radiotherapy Consortium).

\section{Acknowledgements}

The authors acknowledge adjunct professor, Ph.D. Mikko Tenhunen for his help and valuable guidance during the project. The authors also kindly thank head physicists Bjarke Mortensen and Martin Berg and greatly appreciate the collaboration with the Department of Medical physics in Vejle Hospital, Denmark.

\section{References}

[1] Seppala T, Visapaa H, Collan J, et al. Converting from CT- to MRI-only-based target definition in radiotherapy of localized prostate cancer: a comparison between two modalities. Strahlenther Onkol 2015;191:862-8.

[2] Aoyama H, Shirato H, Nishioka T, et al. Magnetic resonance imaging system for three-dimensional conformal radiotherapy and its impact on gross tumor volume delineation of central nervous system tumors. Int J Radiat Oncol Biol Phys 2001;50:821-7.

[3] Khoo VS, Joon DL. New developments in MRI for target volume delineation in radiotherapy. Br J Radiol 2006;79:2.

[4] Rasch C, Barillot I, Remeijer P. Touw A, van Herk M, Lebesque JV. Definition of the prostate in CT and MRI: a multi-observer study. Int J Radiat Oncol Biol Phys 1999;43:57-66.

[5] Hentschel B, Oehler W, Strauss D, Ulrich A, Malich A. Definition of the CTV prostate in CT and MRI by using CT-MRI image fusion in IMRT planning for prostate cancer. Strahlenther Onkol 2011;187:183-90.

[6] Nyholm T, Nyberg M, Karlsson MG, Karlsson M. Systematisation of spatial uncertainties for comparison between a MR and a CT-based radiotherapy workflow for prostate treatments. Radiat Oncol 2009;4:54.

[7] Korhonen J, Kapanen M, Sonke JJ, et al. Feasibility of MRI-based reference images for image-guided radiotherapy of the pelvis with either cone-beam computed tomography or planar localization images. Acta Oncol 2015:54:889-95.

[8] Korhonen J, Kapanen M, Keyrilinen J, Seppl T, Tuomikoski L, Tenhunen M. Absorbed doses behind bones with MR image-based dose calculations for radiotherapy treatment planning. Med Phys 2013;40.

[9] Korhonen J, Kapanen M, Keyrilinen J, Seppl T, Tenhunen M. A dual model HU conversion from MRI intensity values within and outside of bone segment for MRI-based radiotherapy treatment planning of prostate cancer. Med Phys 2014;41.

[10] Korhonen J. Magnetic Resonance imaging -based Radiation Therapy-Methods Enabling the Radiation Therapy Treatment Planning Workflow for Prostate Cancer Patients by Relying Solely on MRI-Based Images Throughout The Process Doctoral Dissertations,. Aalto University Publication Series; 2015. pp. $1-66$.

[11] Dowling JA, Sun J, Pichler P, et al. Automatic substitute computed tomography generation and contouring for magnetic resonance imaging (MRI)-alone external beam radiation therapy from standard MRI sequences. Int J Radiat Oncol Biol Phys 2015;93:1144-53.

[12] Gustafsson C, Korhonen J, Persson E, Gunnlaugsson A, Nyholm T, Olsson LE. Registration free automatic identification of gold fiducial markers in MRI target delineation images for prostate radiotherapy. Med Phys 2017.

[13] Ghose S, Mitra J, Rivest-Hénault D, et al. MRI-alone radiation therapy planning for prostate cancer: automatic fiducial marker detection. Med Phys 2016;43:2218-28

[14] Edmund JM, Nyholm T. A review of substitute CT generation for MRI-only radiation therapy. Radiat Oncol 2017:12.

[15] Jonsson JH, Karlsson MG, Karlsson M, Nyholm T. Treatment planning using MRI data: an analysis of the dose calculation accuracy for different treatment regions. Radiat Oncol 2010;5:62.

[16] Chin AL, Lin A, Anamalayil S, Teo BK. Feasibility and limitations of bulk density assignment in MRI for head and neck IMRT treatment planning. J Appl Clin Med Phys 2014;15:4851.

[17] Guerreiro F, Burgos N, Dunlop A, et al. Evaluation of a multi-atlas CT synthesis approach for MRI-only radiotherapy treatment planning. Phys Med 2017.

[18] Dowling JA, Lambert J, Parker J, et al. An atlas-based electron density mapping method for magnetic resonance imaging (MRI)-alone treatment planning and adaptive MRI-based prostate radiation therapy. Int J Radiat Oncol Biol Phys 2012;83:5.

[19] Edmund JM, Kjer HM, Van Leemput K, Hansen RH, Andersen JA, Andreasen D. A voxel-based investigation for MRI-only radiotherapy of the brain using ultra short echo times. Phys Med Biol 2014;59:7501-19.

[20] Kapanen M, Tenhunen M. T1/T2*-weighted MRI provides clinically relevant pseudo-CT density data for the pelvic bones in MRI-only based radiotherapy treatment planning. Acta Oncol 2013:52:612-8.

[21] Koivula L. Magnetic Resonance Imaging- Based Radiation Therapy Treatment Planning (Master's thesis). University of Helsinki; 2016. p. 1-38. http://hdl. handle.net/10138/161241.

[22] Koivula L, Wee L, Korhonen J. Feasibility of MRI-only treatment planning for proton therapy in brain and prostate cancers: dose calculation accuracy in substitute CT images. Med Phys 2016;43:4634.

[23] Kapanen M, Collan J, Beule A, Seppala T, Saarilahti K, Tenhunen M. Commissioning of MRI-only based treatment planning procedure for external beam radiotherapy of prostate. Magn Reson Med 2013;70:127-35.

[24] Persson E, Gustafsson C, Nordstrom F, et al. MR-OPERA: multicenter/multivendor validation of magnetic resonance imaging-only prostate treatment planning using synthetic computed tomography images. Int J Radiat Oncol Biol Phys 2017.

[25] Nyul LG, Udupa JK, Zhang X. New variants of a method of MRI scale standardization. IEEE Trans Med Imaging 2000;19:143-50.

[26] Robitaille N, Mouiha A, Crepeault B, Valdivia F, Duchesne S. The Alzheimer's disease Neuroimaging Initiative. Tissue-based MRI intensity standardization: application to multicentric datasets. Int J Biomed Imaging 2012;2012:347120.

[27] Hounsfield GN. Computerized transverse axial scanning (tomography). 1. description of system. Br J Radiol 1973;46:1016-22.

[28] Prior P, Chen X, Botros M, et al. MRI-based IMRT planning for MR-linac: comparison between CT- and MRI-based plans for pancreatic and prostate cancers. Phys Med Biol 2016;61:3819-42.

[29] Korhonen J, Kapanen M, Keyrilainen J, Seppala T, Tuomikoski L, Tenhunen M. Influence of MRI-based bone outline definition errors on external radiotherapy dose calculation accuracy in heterogeneous pseudo-CT images of prostate cancer patients. Acta Oncol 2014;53:1100-6.

[30] Siversson C, Nordstrom F, Nilsson T, et al. Technical note: MRI only prostate radiotherapy planning using the statistical decomposition algorithm. Med Phys 2015;42:6090-7.

[31] Kim J, Glide-Hurst C, Doemer A, Wen N, Movsas B, Chetty IJ. Implementation of a novel algorithm for generating synthetic CT images from magnetic resonance imaging data sets for prostate cancer radiation therapy. Int J Radiat Oncol Biol Phys 2015;91:39-47.

[32] Johansson A, Karlsson M, Nyholm T. CT substitute derived from MRI sequences with ultrashort echo time. Med Phys 2011;38:2708-14.

[33] Schneider U, Pedroni E, Lomax A. The calibration of CT hounsfield units for radiotherapy treatment planning. Phys Med Biol 1996;41:111-24.

[34] Kuttner S, Bujila R, Kortesniemi M, et al. A proposed protocol for acceptance and constancy control of computed tomography systems: a nordic association for clinical physics (NACP) work group report. Acta Radiol 2013;54:188-98. 
[35] Vovk U, Pernus F, Likar B. A review of methods for correction of intensity inhomogeneity in MRI. IEEE Trans Med Imaging 2007;26:405-21.

[36] Tustison NJ, Avants BB, Cook PA, et al. N4ITK: improved N3 bias correction. IEEE Trans Med Imaging 2010;29:1310-20.

[37] Gustafsson C, Nordstrom F, Persson E, Brynolfsson J, Olsson LE. Assessment of dosimetric impact of system specific geometric distortion in an MRI only based radiotherapy workflow for prostate. Phys Med Biol 2017;62:2976-89.

[38] Wang H, Balter J, Cao Y. Patient-induced susceptibility effect on geometric distortion of clinical brain MRI for radiation treatment planning on a $3 \mathrm{~T}$ scanner. Phys Med Biol 2013;58:465-77.
[39] Chen Z, Ma CM, Paskalev K, et al. Investigation of MR image distortion for radiotherapy treatment planning of prostate cancer. Phys Med Biol 2006;51:1393-403.

[40] Torfeh T, Hammoud R, Perkins G, et al. Characterization of 3D geometric distortion of magnetic resonance imaging scanners commissioned for radiation therapy planning. Magn Reson Imaging 2016;34(5):645-53.

[41] Kemppainen R, Suilamo S, Tuokkola T, Lindholm P, Deppe MH, Keyrilainen J. Magnetic resonance-only simulation and dose calculation in external beam radiation therapy: a feasibility study for pelvic cancers. Acta Oncol 2017:1-7. 